\title{
Approaches to preventive periodontal care for geriatric patients with somatic disorders
}

\author{
Tatiana Elovikova ${ }^{1}$, Svetlana Sablina ${ }^{1}$, Sergei Grigorjev ${ }^{1}$, Vera Karaseva ${ }^{1}$, and Anatoly \\ Koscheev $^{2}$ \\ ${ }^{1}$ Department of Preventive Dentistry and Propedeutics of Dental Disease, Ural State Medical \\ University, 620028, Repina Str., 3, Ekaterinburg, Russia \\ ${ }^{2}$ Department of Control Systems Modeling, Ural Federal University, 620002, Mira Str., 19, \\ Ekaterinburg, Russia
}

\begin{abstract}
During the last two decades the acute interest in longevity has been kept by the world's scientific community in relation to quality of health of older adults and prevention of emerging diseases including oral disorders in the old age. This article presents the study of oral conditions in 100 patients between the ages of 60 and 69 who were divided into three groups. This study aims to define approaches to preventive periodontal care for elderly patients with comorbidities. The primary group included patients with periodontal disease (PD) and salivary gland dysfunction: Sjogren's disease and syndrome, non-insulin dependent diabetes mellitus, chronic pancreatitis. Two experimental groups included patients with normal salivary gland function and PD as well practically healthy persons without PD and normal salivary gland function. A comparative analysis of the results showed spread of dental caries $(100 \%)$ and periodontal diseases $(80 \%)$. The clear correlation between periodontium complex inflammation and poor oral hygiene is defined, mostly with men $(\mathrm{p}<0.05)$. Structural and optical properties of mixed saliva significantly change subject to periodontium inflammation and xerostomia: severity of xerostomia corresponding to profound hemodynamic and microcirculatory changes causes more intensive structural developmental abnormalities of mixed saliva. The important component of the Periodontal Treatment Protocol is to help older people develop skills and controls of effective thorough tooth brushing.
\end{abstract}

\section{Introduction}

Currently, the steady trends in population aging are becoming apparent in the world due to the increase in number of persons aged 60 and over. The medical discipline of gerodontology is actively developing today, its concepts relating to oral disease prevention and tooth-retention among older adults are mastered [1].

The most prevailing oral disease is periodontitis, an inflammation of the periodontal tissues with destruction - generalized chronic periodontitis. It is the infectious nidus involving serious agents such as A.actinomycetemcomitans, P.gingivalis, Tannerella forsythia, Treponema denticola, the sensibilization source of the body and the key problem 
related to tooth loss by elderly patients causing serious dentinal disorganization not only of dentofacial but cognitive functions of older patients [2,3,4,5].

All these are worsened by various combinations of existing risk factors, somatic and age-related systematic diseases (osteoporosis, osteopenia), dysmetabolism and trophism in elderly people [6,7].

Dentofacial and oral features of patients above 60 years have become subjects to numerous and broad recent researches but specialty doctors as part of their multidisciplinary activities prioritize searching consensus in relation to specific clinical and practical approaches $[8,9]$. Therefore, providing preventive dental care for elderly patients has become an actual problem.

Today, gerodontology is a new discipline which has emerged at the joint of gerontostomatology and periodontology; it addresses the study, treatment and prevention of periodontal pathology in seniors and elders enabling to personify preventive and therapeutic techniques, improve projected tooth-retention and periodontium, thus, enhance the quality of life for people as they age to the utmost [10].

This study aims to define approaches to preventive periodontal care and comorbidity for older people with salivary gland hypofunction.

\section{Materials and Methods}

100 patients at the age from 60 to 69 years old are studied. The patients were divided into three groups. The primary group (50 women, $10 \mathrm{men}$ ) included patients with periodontal diseases (PD) - generalized chronic periodontitis and salivary gland dysfunction: 1.1. Sjogren's disease and syndrome (SD, SS) - 20 persons; 1.2. - non-insulin dependent diabetes mellitus (NIDDM) - 20 persons; 1.3. - chronic pancreatitis (CP) - 20 persons. The background disease - NIDDM, SD and SS, CP - is defined by respective medical specialists (endocrinologists, rheumatologists, gastroenterologists, etc.). Two experimental groups included: K 1 - patients of the same age with normal salivary gland function and PD - 20 persons (13 women, 7 men); 2) practically healthy persons without PD and normal salivary gland function -20 persons ( 14 women, 6 men) applied for oral cavity sanation to the department.

The patients were divided into groups according to age based on the classification of the WHO Regional Office for Europe, where the 60 to 74 year old group is the elderly age; the 75 to 89 year old group is the old age and the 90 year and over group is long-livers. This classification, in our opinion, adequately reflects the situation as reaching the age range of 60 years is considered passing from maturity to old age; it is also caused by physiological body changes and social/psychological processes of personality reorganization [8].

Depending on etiology, pathogeny, clinical/laboratory data, there were defined parenchymal and interstitial types of sialosis in accordance with the commonly used classification [11,12]. To define conditions of the oral cavity and make a diagnosis, all patients underwent the complete examination including clinical, X-ray, laboratory and auxiliary examination methods among those interrogation, visual evaluation, palpation of regional globate glands and salivary glands with definition of the hygiene index (HI, GreenVermillion). The periodontal index (PMA, Parma, 1960; PI Russell, 1956) was assessed; the depth of periodontal pockets, tooth mobility, the level of gingival recession were measured; DMFT index and DMF calculated per tooth surface were defined. The condition and the structure of the bone tissue of the alveolar bone were assessed using X-ray. Salivary glands were examined with the following methods: sialometry, the qualitative analysis of secretions (color, transparency, inclusions, viscosity, $\mathrm{pH}$ ), clinical examination and stomatoscopy of minor salivary glands: swelling, hyperemia, hyperplasia; functional activity of salivary glands. Laboratory and auxiliary examinations included: clinical blood 
test, common urine analysis, blood chemistry (glucose levels measured); as well consultations of therapists, endocrinologists, rheumatologists and other medical specialists. Structural and optical properties of mixed saliva were studied by polarization microscopy which enabled to register metabolic disorders and structural changes in molecular complexes of mixed saliva; mineralizing potential of mixed saliva and type of microcrystallization of mixed saliva were assessed when viewing the total area and the dried drop of mixed saliva depending on five types found [11]. Absorption efficiency of microorganisms through oral mucosal epithelial cells was examined by the modified method of N.Danilevsky, T.Belenchuk [12]. Dental patient medical records are completed [13].

Statistical analysis of the results is done with PC Statistica 6.0 software package. The data are presented in the form of arithmetic means and the standard error of the mean $(\mathrm{M} \pm \mathrm{m})$. Reliability of the difference score is estimated by Student's t-distribution. Difference estimations are precise at $\mathrm{p}<0.05[14,15]$.

\section{Results}

Patients of group 1.1 complained of dry mouth, enlarged salivary glands, whereof parenchymal sialosis and bilateral enlargement of salivary glands (mainly, parotid) are diagnosed in $100 \%$ of cases. The total of 20 persons is diagnosed with initial xerostomia (Fig.1). When viewing the oral mucosa - lips, cheeks, palate, tongue, the floor of the mouth, the following was found: thinning and flattening of the papillae, lingual folds. Unsatisfactory $\mathrm{HI}$ is observed. With initial xerostomia, parotid salivary gland enlargement is seen in 6 persons. Mixed saliva indicates normal values of $\mathrm{pH}$ and viscosity. The $25 \%$ decrease is in functional activity of salivary glands. Partial tooth loss is observed for every patient. DMFT index reached 32, DMF calculated per tooth surface is $46, \mathrm{~K}$-component (caries) is the dominant component of the index. The patients did not mention bleeding or other complaints "concerning gums" except for biting and chewing food which can be challenging as the food can "become stuck in the teeth", underneath dentures as teeth keep constantly breaking. PMA indexes indicate inflammation of the gingival margin. For SS patients, the slight and moderate periodontitis is found. The type 4 of microcrystallization of mixed saliva, the score of 1 (Fig.2), the lower mineralizing potential (1.1-2). Absorption of microorganisms through oral mucosal epithelial cells: category 4 cells mainly prevail for periodontitis patients with SS. These prove deficiency of nonspecific resistance of the oral cavity and the systematic disorder of the exocrine gland. Structural and optical properties of oral fluids: needle-shaped crystals, large dendrites, layered spheroliths, confocal domains. For SD, SS patients, the correlation of sialosis is observed with duration and degree of xerostomia. The results of examination prove the direct correlation between severity of SD, SS and xerostomia severity $(r=+1.0)$; as well PD is correlated with duration of SD $(r=+$ $0.4)$. 


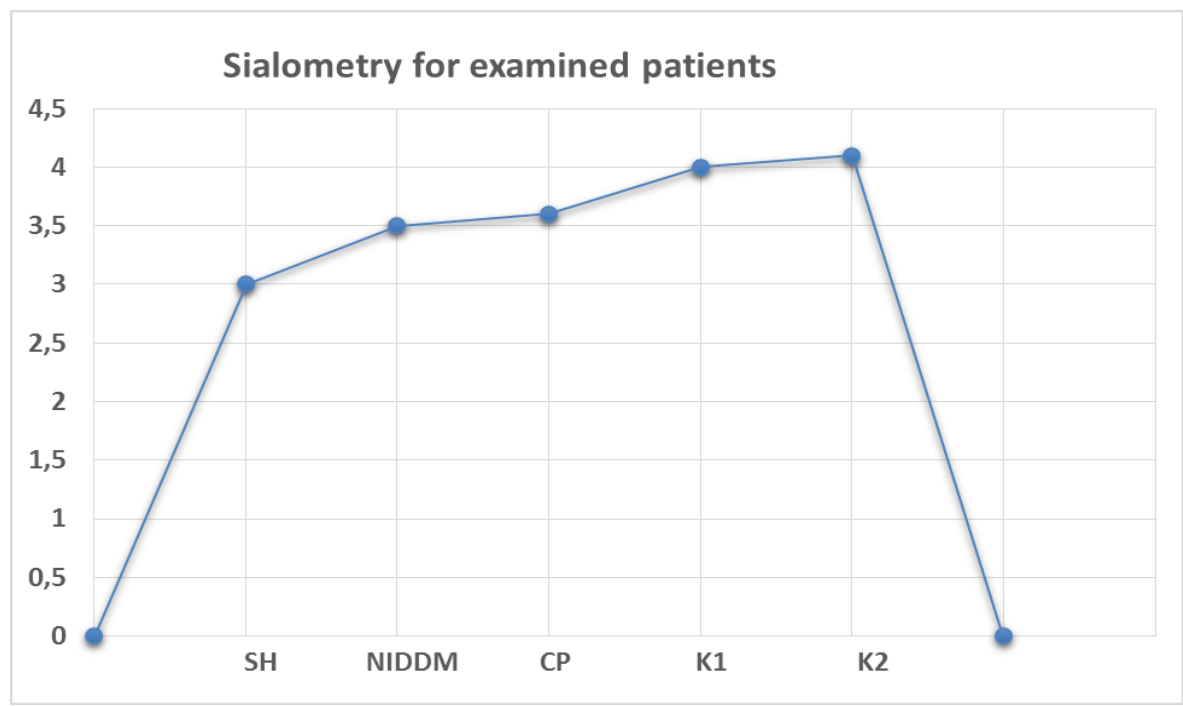

Fig. 1. Sialometry for examined patients

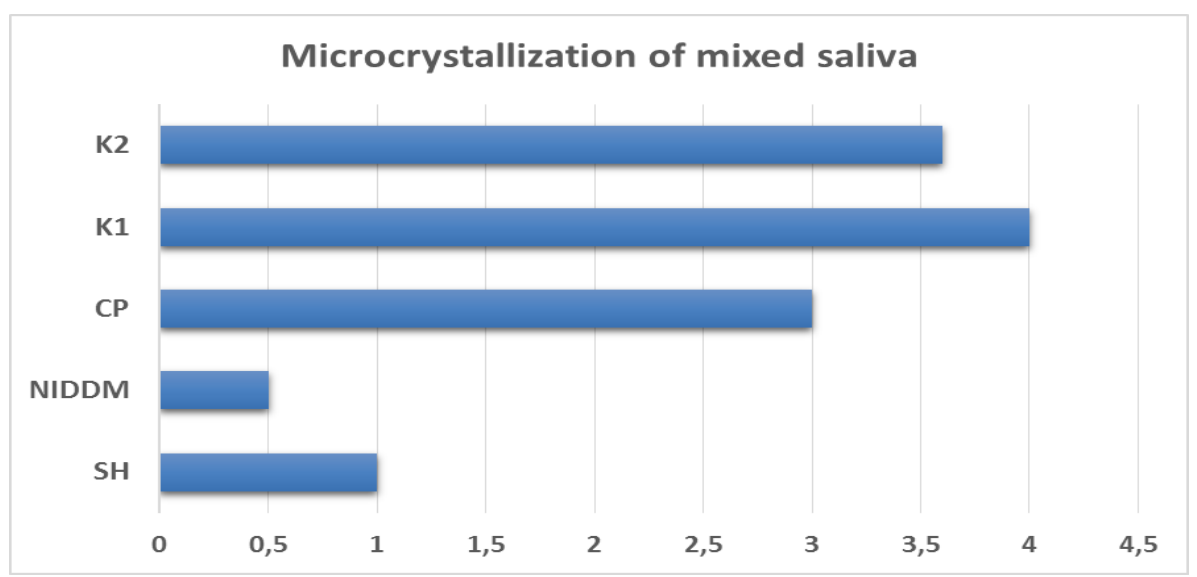

Fig. 2. Microcrystallization of mixed saliva for examined patients (score)

With carbohydrate metabolism disorders, NIDDM, salivation and quality of mixed saliva become less as well. NIDDM patients complained of dry mouth which increased when the general condition worsened. Interstitial sialosis (IS) is observed in $100 \%$ of cases and associated with soft painless bilateral swelling in the area of parotid salivary glands. All patients are diagnosed with initial xerostomia irrespective of diabetes duration (Fig.1). IS is long-standing without any inflammatory signs. Mixed saliva is foamy with increased viscosity because of worsening of the general condition. The $\mathrm{pH}$ value is slightly acidic (average of 6.2). Functional activity of salivary glands is reduced by $20 \%$.

Periodontal tissues evidence progressive inflammatory destruction in $100 \%$ of cases (increased average PMA up to $85.5 \% \pm 5,5 \%$; PI - from 3.5 to $5.5 \pm 0.5$ ). The direct correlations between PD and NIDDM duration $(\mathrm{r}=+0.623)$, NIDDM severity, xerostomia severity and PD severity (respectively, $r=+1.0$ and $r=+0.424$ ) are confirmed.

Changes of types 4-5 of mixed-saliva microcrystallization are observed (the score of 0.5; (Fig.2), the mineralizing potential is low. Absorption of microorganisms through oral mucosal epithelial cells: with worsened periodontal condition, absorption activity of epithelial cells tends to be slower which demonstrates the loss of the intrinsic burrier 
function of cells and impairment of morphofunctional characteristics, and consequently, reduced nonspecific resistance of the oral cavity. Structural and optical properties of mixed saliva: large dendrites, needle-shaped and large layered crystals. All patients with NIDDM are diagnosed with chronic periodontitis. With slight and moderate generalized chronic periodontitis in patients with NIDDM, the typical traditional clinical picture is observed. Mixed-saliva microcrystallization (the score of 0 ) is Type 5, mineralizing potential is very low. Absorption of microorganisms through oral mucosal epithelial cells: there is some tendency to reduce absorption efficiency of microorganisms through oral mucosal epithelial cells proving impairment of morphofunctional characteristics. Structural and optical properties of oral fluids: needle-shaped textures, dendrites, spheroliths.

$50 \%$ of those examined with CP periodically complained of dry mouth. Patients had unsatisfactory HI ( $2.4 \pm 0.6$ points). It is observed that dental tartar and plague is formed rapidly. Conditions of periodontal tissues show progressive inflammatory destruction in $100 \%$ of cases (PMA on average is increased to $75.5 \% \pm 7.5 \%$; HI - from 3.54 to $4.9 \pm$ 0.5). The direct correlation between $\mathrm{PD}$ and $\mathrm{CP}$ duration $(\mathrm{r}=+0.453)$, between $\mathrm{CP}$ severity, xerostomia severity and severity of periodontal involvement (respectively, $r=+$ 0.476 and $\mathrm{r}=+0.453)$ is determined. Some changes of type 2 of mixed-saliva microcrystallization (the score of 3; (Fig. 2) are observed, the mineralizing potential is high. Absorption of microorganisms through oral mucosal epithelial cells: a slight tendency for moderate impairment of the barrier function and deficiency of nonspecific resistance of the oral cavity. Structural and optical properties of mixed saliva: dendrites and spheroliths, different forms of LC-aggregates.

\section{Discussions}

Based on the comparative study of collected clinical and laboratory data, we could define the most significant differential and clinical features of PD with xerostomia. At the same time, the extent of xerostomia is not the dominant cause of PD. Thus, with SH, the extremely severe autoimmune disorder, periodontitis is just a "quiet" disease caused by local irritations. The high $\mathrm{pH}$-value of mixed saliva is found. It should be noted that with $\mathrm{SH}$, periodontium is not the potential target organ.

The more long-term and severe NIDDM is, microbiota resistance of oral mucosa is significantly lower, reactive conditions of oral mucosa, characteristics of periodontal tissue and mixed saliva are decreased accelerating destruction of periodontal tissues. Started with physiological strain, the process leads to broken adaptation and PD appears more severely on the background of the worst oral hygiene of patients with NIDDM $[16,17,18,19]$.

With xerostomia, the initial protection (mixed saliva) is practically excluded: PD is found in $100 \%$ persons with decreased secretary function of salivary glands. In mixed saliva samples of patients with xerostomia, we found four main types of textures such as dendrites, spheroliths, needle-shaped crystals and layers. Their dynamic composition correlates with the clinical picture and reflects physicochemical changes in mixed saliva and, in general, accurately corresponds to severity of PD with xerostomia. Severity of xerostomia causes intensive formation of pathological types of mixed saliva components: formed dendrite crystals trace activation of reparative processes, formed needle-shaped crystals, spheroliths and layers are connected with body destruction processes. Therefore, salivary gland hypofunction is one of the steps of the mechanism of PD development because sialosis destroys the initial protection (mixed saliva).

The results of the study have shown: spread of dental caries for all patients is $100 \%$. High spread and intensity of periodontal pathology increasing with age, periodontal pockets $5.5 \mathrm{~mm}$ or more in depth are for $80 \%$ of examined patients. 
The clear correlation of periodontium inflammation with poor oral hygiene is defined, mainly with men $(\mathrm{p}<0.05)$.

The main characteristic of the periodontal disease in the elderly is smoldering generalized periodontitis characterized by "erased" clinical implications in the background of severe periodontal destruction processes. The above evidences the high need of dental care for all examined patients and the preventive periodontal care program for the elderly population [20].

This program shall include: compliance with Periodontal Treatment Protocol for patients with periodontal disease, specifics of professional oral hygiene and gerontostomatological aspects, case follow-up and supporting therapy, quality control of personal hygiene, motivational talks, dental (and periodontal) rehabilitation of elderly patients. Therefore, the mentioned points aim to make actual the following key goal: improve the quality and expectancy of life of geriatric patients [21].

\section{Conclusions}

1. Elderly persons with decreased secretary function of salivary glands show inflammatory PD in $100 \%$ of cases.

2. PD in geriatric patients with salivary gland hypofunction is not a specific disease, the extent of clinical features does not depend on xerostomia degree.

3. Salivary gland hypofunction is one of the steps of PD mechanism. Xerostomia is often a complicating factor causing inadequate protective response of the body.

4. Structural and optical properties of mixed saliva undergo major changes as the result of inflammatory PD and xerostomia: severity of xerostomia corresponding to major hemodynamic and microcirculatory changes causes more intensive formation of pathological types of mixed saliva components.

5. Most of those examined were not informed about the concept of preventive dentistry at a young age and therefore they have no respective inclinations. The important component of the Periodontal Treatment Protocol is to help older people develop skills and controls of effective thorough tooth brushing.

\section{References}

1. M.S. Tonetti, P. Bottenberg et al., Clinical Periodontology, 44(S18), 135-144 (2017)

2. L.J. Dominguez, M. Barbagallo, Current Opinion in Clinical Nutrition \& Metabolic Care, 20(1), 61-68 (2017)

3. Z. Seraj, Dana Al-Najjar et al., Int. Journal of Dentistry, 2017(4) 1-6 (2017)

4. W.L Fang., M.J. Jiang, Y.M. Wei, S.N. Fan, et al., BMC Psychiatry, 18, 345 (2018)

5. V.V. .Lobeiko, A.K. Iordanashvili, V.F. Chernysh, Periodontology, 22(3(84)), 29-32 (2017)

6. M. Bernal, M. Elenkova, J. Evensky, S. H.Stein, Current Oral Health Reports, 5, 26 (2018)

7. T.-H. Lin, Ch.-Ch. Lung, et al., Medicine (Baltimore), 94, 7 (2015)

8. F. De Angelis, S. Basisi, et al., Journal of Immunopathology and Pharmacology, JanFeb (2018)

9. K. Oluwagbemigun, T. Dietrich, N. Pischon, PLoS, One, 10, 5 (2015)

10. S.V. Kusnetsov, Y.A. Dzevichek, O.V. Shevchenko, Electronic scientific journal APRIORI. Series: Natural and Engineering Science, J.5 (2016)

11. T.M. Elovikova, S.S. Grigoriev, Sialology in therapeutic dentistry, 192 (2018) 
12. T.M. Elovikova, S.S. Grigoriev, Saliva as a biological fluid and its role in oral health, $136(2018)$

13. T.M. Elovikova, A.V. Troshunin, E.E. Zhukova, Z.E. Ozhgihina, Periodontology, 3, 5154 (2013)

14. T.M. Elovikova, Actual Problems in Dentistry, 5, 8-11 (2013)

15. T.M. Elovikova, V.V. Karaseva, V.S. Molvinskikh, Y.S. Skurikhina, A.S. Koshcheev, Actual Problems in Dentistry, 15(4), 44-49 (2019)

16. S.S. Grigoriev, P.N.Kudinov, A.V.Burlak, Ural Medical Journal, 12(180), 18-25 (2019)

17. S.V. Krainov, V.F. Mikhalchenko, A.T. Yakovlev, A.N. Popova, I.V. Firsova, Success of Modern Science and Education, 3(9), 167-169 (2016)

18. P. Abdul Rezak, K.M. Jose Richard, R.P. Thankachan, K.A. Abdul Hafiz, K. Nanda Kumar, K.M. Samer, J. Int. Oral Health, 6(6), 110-116 (2014)

19. V. Murthy, Y.Vaithilingam, S.Shivaprasad, N.Yadav, S. Dayalan, J. Indian Prosthodont. Soc., 15(2), 119-124 (2015)

20. Y. Watanabe, K. Okada, M. Konoda, T. Matsushita, S. Nakazava, Y. Yamazaki, Geriatrics\&Gerentology International, 20(4) (2020)

21. S. Morishita, Y. Watanabe, Y. Ohara, A. Edahiro, E. Sato, T. Suga, H. Hirano, Factors associated with older adults need for oral hygiene management by dental professionals, 16(8) (2015) 